\title{
The preferential contraction ratios of transversus abdominis on the variations of knee angles during abdominal drawing-in maneuver in wall support standing
}

\author{
Young-In Hwang' ${ }^{1}$ Jwa-Jun Kim², Du-Jin Park ${ }^{3, *}$ \\ 'Department of Physical Therapy, Dong-A University Hospital, Busan, Korea \\ 2Department of Physical Therapy, Choonhae College of Health Sciences, Ulsan, Korea \\ ${ }^{3}$ Department of Physical Therapy, College of Health Medicine, Kaya University, Gimhae, Korea
}

The objective of this study was to determine appropriate knee angles for the abdominal drawing-in maneuver (ADIM) through evaluation of changes in contraction ratios of the abdominal muscles and activity of quadriceps muscle in relation to changes in knee angles occurring while the ADIM is performed in the wall support standing (WSS). 20 subjects performed the ADIM at different knee angles $\left(0^{\circ}, 20^{\circ}, 40^{\circ}, 60^{\circ}\right)$ in random order, standing at a point 6 inches away from the wall with the spine maintained in the neutral position. The WSS with knee flexion at $20^{\circ}$ showed significantly higher preferential contraction ratio (PCR) of transversus abdominis (TrA) compared to other positions $\left(0^{\circ}, 40^{\circ}, 60^{\circ}\right)$. Therefore, performing the ADIM in the WSS with knee flexion at $20^{\circ}$ appears to be the most appropriate position for TrA PCR.

Keywords: Wall support standing, Knee angles, Abdominal drawing-in maneuver, Transversus abdominis

\section{INTRODUCTION}

Lumbar stabilization exercise is gradually becoming more popular as a treatment for athletes and people with low back pain (LBP). The abdominal drawing-in maneuver (ADIM) is attracting great attention as a sort of lumbar stabilization exercise that can improve the core system that is responsible for spinal stability. The ADIM is effective in recovering the motor control of the transversus abdominis ( $\operatorname{Tr} A$ ) while minimizing the contraction of the abdominal muscles located in the superficial layer (Springer et al., 2006), and relieving pain and functional disorder in the lumbar and pelvic regions (Stuge et al., 2004).

Because of these advantages, the ADIM is frequently used as a basic exercise element in lumbar stabilization training programs (O'Sullivan et al., 1998). Recently, studies that applied the ADIM together with diverse exercises were showed the effects of ADIM (Bjerkefors et al., 2010; Park et al., 2011). Bjerkefors et al. (2010) reported increasing selective activity of the $\operatorname{Tr} A$ from the rectus abdominis (RA) located in the superficial layer while 5 different trunk stabilization exercises performed with the ADIM. In addition, Park et al. (2010) also reported that when people with lumbar extension rotation syndrome performed the ADIM with knee flexion in a prone position, their LBP, pelvic movements and trunk extensor activity decreased while the activity of their knee flexors increased. As such, the ADIM is applied in combination with diverse exercises as a way to increase the effects of exercises.

As starting positions to perform the ADIM, clinical experts commonly recommend crook lying, prone lying, four points kneeling and wall support standing (WSS). Although all these positions are effective in learning the ADIM, Mew (2009) suggested that performing the ADIM in standing position was effective to increase of TrA activity rather than lying positions. O'Sullivan (2000) also recommended performing the ADIM first in weight-bearing positions such as standing positions and performing it in non-weight-
${ }^{*}$ Corresponding author: Du-Jin Park

Department of Physical Therapy, College of Health Medicine, Kaya University, 208 Samgye-ro, Gimhae 609-757, Korea

Tel: +82-55-330-1000, Fax: +82-55-331-0112, E-mail: djpark35@kaya.ac.kr

Received: February 25, 2014 / Accepted: April 16, 2014
This is an Open Access article distributed under the terms of the Creative Commons Attribution Non-Commercial License (http://creativecommons.org/licenses/by-nc/3.0/) which permits unrestricted non-commercial use, distribution, and reproduction in any medium, provided the original work is properly cited. 
bearing positions such as crook lying, prone lying or four point kneeling positions only when the ADIM could not be performed properly in weight-bearing positions. Furthermore, Akuthota and Nadler (2004) also stated that people with LBP perform and endure exercise in advanced positions such as standing positions was desirable for functional improvement.

Among the four starting positions to perform the ADIM, the WSS is more functional compared to other starting positions. The WSS keeps the spine neutral with the heel 6 inches away from the wall (Norris, 2001). However, concrete descriptions of this position are insufficient, making it difficult to apply this position consistently to all subjects. In particular, as there is no concrete description of knee angles, different knee angles are applied depending on subjects' heights and convenience, so that the loads imposed on the lower extremities and lumbar and pelvic regions' positions are different. Therefore, studies are necessary to determine knee angles that can be consistently applied to all subjects so that they can perform the ADIM successfully. The objective of the present study was to determine appropriate knee angles for the ADIM through evaluation of changes and correlations in contraction ratios (CRs) of the abdominal muscles and activity of quadriceps in relation to changes in knee angles occurring while the ADIM is performed in the WSS.

\section{MATERIALS AND METHODS}

\section{Subjects}

The present study was conducted among healthy males who voluntarily agreed to participate in the study after hearing explanations about the purpose and process of the study. Those who had experienced LBP within the past six months, those who had pain while performing the ADIM, those who had any deformity in their spine or lower extremities, those who had received any surgical intervention, those who had received ADIM training and those who could not perform the exercise in the present study were excluded. The final number of subjects who were selected based on the aforementioned criteria and underwent all processes in the present study was 20; their mean age, height, weight and BMI were $24.43 \pm 4.09 \mathrm{yr}, 172.09 \pm 5.12 \mathrm{~cm}, 64.48 \pm 5.21 \mathrm{~kg}$ and $21.75 \pm 1.03$, respectively. For study consistency, all subjects were right-handed.

\section{ADIM protocol}

All the subjects learned the ADIM using a pressure biofeedback unit (PBU; Fig. 1). First, each subject was instructed to take a su-

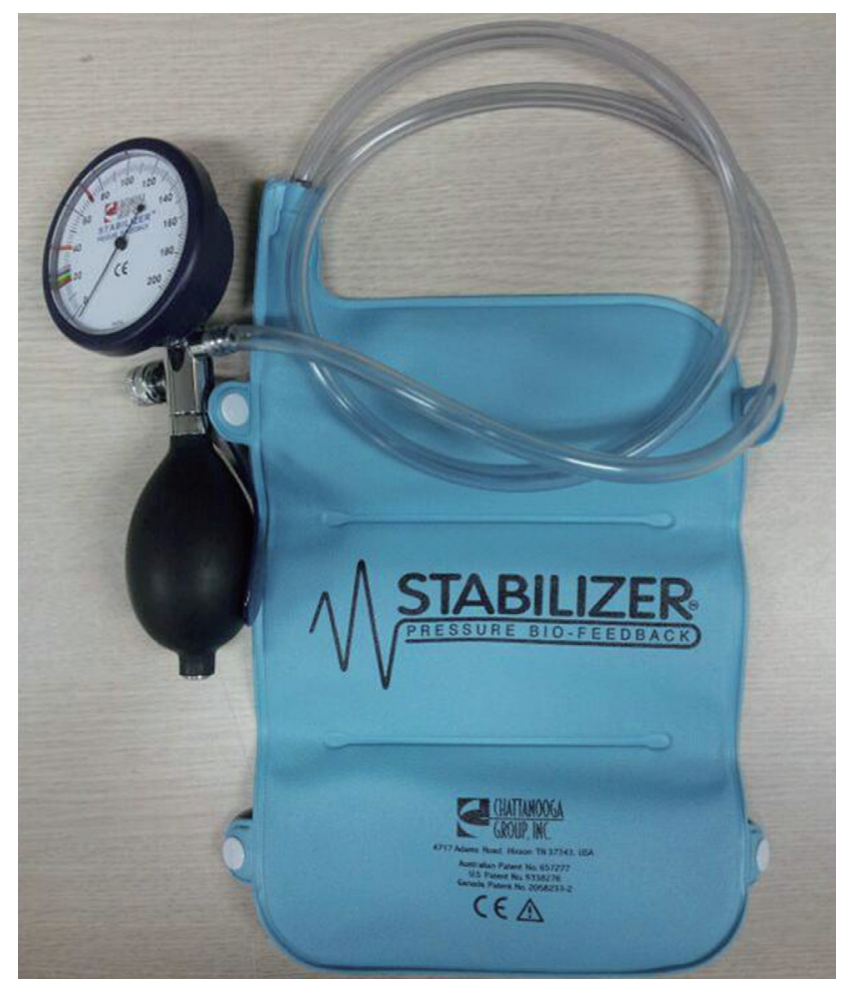

Fig. 1. Pressure biofeedback unit.

pine position with his knees bent to $90^{\circ}$, and a PBU (Chattanooga Group Inc., Hixson, TN37343, USA) was placed between the subject's lumbar lordosis. Then, the bulb was inflated to 40 $\mathrm{mmHg}$ and increased at a pressure of $0-2 \mathrm{mmHg}$ (Park and Lee, 2013) while the subject was instructed on the ADIM as follows. For $30 \mathrm{~min}$, the subject was instructed to slowly draw in the low abdomen without moving the spine, ribs or pelvis while contracting the pelvic floor simultaneously.

The foregoing two activities were performed together because the acts of the lateral abdominal muscles and the pelvic floor are related to the intra-abdominal pressure control mechanism (Norris, 2001), and the contraction of the pelvic floor can help the performance of ADIM (Critchley, 2002; Norris, 2001). As a method to contract the pelvic floor, we used the "lifting the penis" method which is useful for men rather than "stop your flow of urine" or "gripping lower abdomen" (Norris, 2001). Only those who accurately performed the ADIM along with the contraction of the pelvic floor using a PBU in a supine position were instructed to perform the ADIM in WSS. Because the degree of upper extremity movements in WSS may affect the activity of the TrA (Park and Lee, 2010), the upper extremities were placed in gentle contact with the trunk to control the movements.

In addition, the chin was gently drawn to get the head contact 
with the wall to control the movements. Then, the subjects performed the ADIM at different knee angles $\left(0^{\circ}, 20^{\circ}, 40^{\circ}, 60^{\circ}\right)$ in random order standing at a point 6 inches away from the wall with the spine maintained in the neutral position. To verify the accuracy of the PBU, a weight of $4.54 \mathrm{~kg}$ was placed on each PBU, and PBUs were observed for $24 \mathrm{~h}$ to use those that showed a pressure decrease of no more than $0.5 \mathrm{mmHg}$ (von Garnier et al., 2009).

\section{Measurement equipments and methods}

A Sonoace X4 (Medison, Korea) ultrasonography instrument was used to measure the thicknesses of the $\operatorname{Tr} A$, the internal oblique (IO) and the external oblique (EO), and a 7.5 MHz linear transducer was used in the measurement. The transducer was transversely placed on the middle abdominal region between the border of the 11th costal cartilage and the iliac crest (Teyhen et al., 2005). To control the effects of breathing, all data were collected at the ends of expiratory phases. The thicknesses of the abdominal muscles were measured at rest and then while the ADIM was being performed. The following equations were used to identify the CRs of the abdominal muscles (Mannion et al., 2008; Manshadi et al., 2011; Teyhen et al., 2005).

$\operatorname{Tr} A C R=\operatorname{Tr} A$ thickness contracted $/ \operatorname{Tr} A$ thickness at rest

$\mathrm{EO} C \mathrm{CR}=\mathrm{EO}$ thickness contracted/EO thickness at rest

$\mathrm{IO}+\mathrm{EO} \mathrm{CR}=\mathrm{IO}+\mathrm{EO}$ thickness contracted/IO $+\mathrm{EO}$ thickness at rest
$\operatorname{Tr} \mathrm{A}$ PCR $=(\operatorname{Tr} \mathrm{A}$ contracted $/ \operatorname{Tr} \mathrm{A}+\mathrm{IO}+\mathrm{EO}$ contracted $)-(\operatorname{Tr} \mathrm{A}$ at rest/TrA $+\mathrm{IO}+\mathrm{EO}$ at rest)

For the surface electromyogram (EMG), MP150WSW (BIOPAC System Inc., USA) was used to measure the muscle activities of vastus medius $(\mathrm{VM})$, rectus femoris $(\mathrm{RF})$ and vastus lateralis (VL) with a bandwidth of $20-450 \mathrm{~Hz}$ and a sampling ratio of $1,000 \mathrm{~Hz}$. To normalize the surface EMG amplitude of quadriceps muscle in this study, all the subjects maintained against manual resistance in manual muscle testing position while maximal voluntary contraction (MVC) was measured. The subjects performed three MVC for 3 sec separated by 2-min rest.

The electrodes were attached to the VM, RF, and VL. The electrodes were attached to $20 \%$ distance from the medial joint line of the knee to the ASIS for VM, 50\% distance ASIS to superior border of patella for RF, $25 \%$ distance from the lateral joint line of the knee to the ASIS for VL (Hubley-Kozey et al., 2013; Rutherford et al., 2011). Each ADIM on the variations of knee angles was performed for $5 \mathrm{sec}$ and the magnitude of the signal from quadriceps muscle was used for 3 sec in this study, omitting the first $1 \mathrm{sec}$ and the last $1 \mathrm{sec}$. Thicknesses of abdominal muscles were measured at $3 \mathrm{sec}$. All subjects performed ADIM in WSS with keeping the neural position of foot ( $7^{\circ}$ toe out).

\section{Statistical analysis}

One-way ANOVA was used to compare changes in the CRs of the abdominal muscles and the activity of quadriceps on variations of knee angles. Scheffe's multiple comparison analysis was used for post-hoc analysis. The Pearson's correlation coefficient was used to examine the correlation between CRs of abdominal muscles and activity of quadriceps on the variations of knee angles during ADIM in WSS. We used the Statistical Package of the Social Sciences version 12.0 (SPSS Inc., Chicago, IL, USA) to analyze the data and set the significance level $\alpha$ to 0.05 . In addition, inter-measurer reliability of measured muscle thickness values was tested using the ICC.

\section{RESULTS}

Table 1 lists the CRs of abdominal muscles on the variations of knee angles during ADIM in WSS. The TrA CRs were significantly higher knee flexion at $60^{\circ}$ than at $0^{\circ}$. The $\mathrm{EO}$ and $\mathrm{IO}+\mathrm{EO}$ CRs were significantly higher knee flexion at $60^{\circ}$ compared to other positions. However, the TrA PCRs were significantly higher knee flexion at $20^{\circ}$ than other positions.

Table 2 lists the activity of quadriceps muscle on the variations of knee angles during ADIM in WSS. As knee angles increased,

Table 1. The contraction ratios of abdominal muscle on variations of knee angles during ADIM in wall support standing $(n=20)$

\begin{tabular}{|c|c|c|c|c|c|c|}
\hline & \multicolumn{6}{|c|}{ Knee flexion degree $\left({ }^{\circ}\right)$} \\
\hline & $0^{\circ}$ & $20^{\circ}$ & $40^{\circ}$ & $60^{\circ}$ & $F$ & $P$ value \\
\hline TrACR & $1.58 \pm 0.13^{\mathrm{a})}$ & $1.67 \pm 0.13^{\mathrm{a}, \mathrm{b}}$ & $1.68 \pm 0.16^{\mathrm{a}, \mathrm{b}}$ & $1.77 \pm 0.16^{b)}$ & 5.161 & 0.003 \\
\hline EOCR & $1.15 \pm 0.07^{a, b)}$ & $1.10 \pm 0.06^{a)}$ & $1.19 \pm 0.06^{b l}$ & $1.31 \pm 0.16^{c)}$ & 16.128 & 0.000 \\
\hline IO+EO CR & $1.14 \pm 0.05^{a, b)}$ & $1.10 \pm 0.07^{a)}$ & $1.20 \pm 0.06^{b /}$ & $1.29 \pm 0.10^{c)}$ & 25.605 & 0.000 \\
\hline TrA PCR & $0.05 \pm 0.01^{a)}$ & $0.07 \pm 0.01^{b)}$ & $0.05 \pm 0.01^{a}$ & $0.05 \pm 0.01^{a)}$ & 8.794 & 0.000 \\
\hline
\end{tabular}

a,b,c) Values within a column with different superscripts letters are significantly different each groups $(P<0.05)$. CR, contraction ratio; PCR, preferential contraction ratio. 
Table 2. The activity of quadriceps muscle on variations of knee angles during ADIM in wall support standing $(n=20)$

\begin{tabular}{|c|c|c|c|c|c|c|}
\hline & & & Knee & gree $\left({ }^{\circ}\right)$ & & \\
\hline & $0^{\circ}$ & $20^{\circ}$ & $40^{\circ}$ & $60^{\circ}$ & $F$ & $P$ value \\
\hline VM & $7.17 \pm 1.85^{a)}$ & $19.36 \pm 3.89^{b)}$ & $28.83 \pm 3.90^{c)}$ & $40.88 \pm 4.49^{d)}$ & 303.020 & 0.000 \\
\hline $\mathrm{RF}$ & $4.59 \pm 1.39^{a)}$ & $11.35 \pm 2.14^{b l}$ & $17.18 \pm 2.39^{c)}$ & $25.19 \pm 2.75^{d)}$ & 309.187 & 0.000 \\
\hline VL & $6.85 \pm 1.52^{\mathrm{a})}$ & $17.64 \pm 3.30^{b)}$ & $28.00 \pm 3.68^{c)}$ & $39.21 \pm 4.24^{d)}$ & 344.488 & 0.000 \\
\hline
\end{tabular}

a, b,c, d) Values within a column with different superscripts letters are significantly different each groups $(P<0.05)$. VM, vastus medius; $\mathrm{RF}$, rectus femoris; $\mathrm{VL}$, vastus lateralis.

Table 3. The relationship between contraction ratios abdominal muscle and activity of quadriceps muscle on variations of knee angles during ADIM in wall support standing $(n=20)$

\begin{tabular}{|c|c|c|c|c|c|c|c|c|}
\hline & KFD & TrACR & EOCR & IOEO CR & TrA PCR & VM & $\mathrm{RF}$ & $V L$ \\
\hline KFD & 1.00 & $0.396^{*}$ & $0.513^{*}$ & $0.600^{*}$ & -0.109 & $0.960^{*}$ & $0.960^{*}$ & $0.965^{*}$ \\
\hline TrA CR & & 1.00 & $0.436^{*}$ & $0.414^{*}$ & $0.577^{*}$ & $0.355^{*}$ & $0.371^{*}$ & $0.358^{*}$ \\
\hline EO CR & & & 1.00 & $0.652^{*}$ & $-0.351^{*}$ & $0.469^{*}$ & $469 *$ & $491^{*}$ \\
\hline IOEO CR & & & & 1.00 & $-0.357^{*}$ & $0.576^{*}$ & $0.557^{*}$ & $0.579^{*}$ \\
\hline TrA PCR & & & & & 1.00 & -0.127 & -0.089 & -0.144 \\
\hline VM & & & & & & 1.00 & $0.962^{*}$ & $0.981^{*}$ \\
\hline $\mathrm{RF}$ & & & & & & & 1.00 & $0.951 *$ \\
\hline$V L$ & & & & & & & & 1.00 \\
\hline
\end{tabular}

${ }^{*} P<0.05$. KFD, Knee Flexion degree $\left({ }^{\circ}\right)$; CR, contraction ratio; PCR, preferential contraction ratio; VM, vastus medius; RF, rectus femoris; VL, vastus lateralis.

the activity of quadriceps muscles were shown to be larger significantly.

Table 3 lists the correlation between the CRs of abdominal muscles, the activity of quadriceps muscle and knee angles. The knee angles showed significant correlation with the CRs of abdominal muscles and the activity of quadriceps muscles except the TrA PCRs. The reliability values for TrA, IO and EO were 0.93, 0.84 , and 0.88 respectively.

\section{DISCUSSION}

In the present study, the $\mathrm{CR}$ of $\operatorname{Tr} \mathrm{A}$ was significantly lower in WSS with knee flexion at $0^{\circ}$ compared to knee flexion at $60^{\circ}$. Although there was no significant difference among other positions $\left(0^{\circ}, 20^{\circ}, 40^{\circ}\right.$ or $\left.20^{\circ}, 40^{\circ}, 60^{\circ}\right)$, the CRs of $\operatorname{Tr} A$ were shown to be larger as knee angles increased. In general, the $\operatorname{Tr} A$ acts at the beginning with tonic activities regardless of the direction of forces acting on the spine (Hodges and Richardson, 1997). The WSS with knee flexion at $0^{\circ}$, loads imposed on the spine should decrease compared to other positions because of support by the knee hyperextension; thus, the tonic activity of the TrA must be relatively low.

On the other hand, as knee flexion increases, the support by the knee skeletal system decreased and the activity of quadriceps increased. In other words, as the difficulty of positions increase, the tonic activity of the TrA will increase to support the spine. In a previous study (Ainscough-Potts et al., 2006), the contraction of the $\operatorname{Tr} A$ increased as the difficulty of positions increased because of instability in position. In addition, as knee flexion increases, the activity of $\operatorname{Tr} A$ that serves direct roles for the stability of the sacroiliac joint and the lumbar and pelvic regions (Richardson et al., 2002) should increase. It is considered that when the ADIM was performed with the increased TrA's postural tone as such, the excitation of the TrA's motor neuron pool and the TrA's ability to mobilize motor neurons increased further.

To perform the ADIM successfully, increasing $\operatorname{Tr} A$ activity is important with minimized activities of the IO and the EO located more superficially (Teyhen et al., 2005). However, in the present study, among three positions $\left(20^{\circ}, 40^{\circ}, 60^{\circ}\right)$ that showed larger $\operatorname{TrA}$ CRs than knee flexion at $0^{\circ}$, two positions $\left(40^{\circ}, 60^{\circ}\right)$ showed significantly larger EO and IO+EO CRs compared to other positions $\left(0^{\circ}, 20^{\circ}\right)$. That is, as knee flexion increased over $40^{\circ}$, not only $\operatorname{TrA}$ CRs increased, but also EO and IO+EO CRs increased. Because of these results, the TrA PCRs decreased. This indicates that EO $(r=-0.351, P=0.001)$ and IO+EO CRs $(r=-0.357$, $P=0.001$ ) were negative correlation with TrA PCR. Additionally, the knee angles showed significant correlation with the activity of quadriceps muscle and CRs of abdominal muscles. Like these results, although performing ADIM in WSS with increased knee flexion may be effective for strengthening of quadriceps and ab- 
dominal muscles, these conditions aren't suitable for increasing TrA PCR.

The WSS with knee flexion at $20^{\circ}$ did not show any significant differences from the two positions $\left(40^{\circ}, 60^{\circ}\right)$ that showed large $\operatorname{TrA}$ CRs than knee flexion at $0^{\circ}$, but showed significant decreases $\mathrm{EO}$ and IO+EO CRs compared to the two positions. As a result, this position showed significantly higher TrA PCRs compared to other positions $\left(0^{\circ}, 40^{\circ}, 60^{\circ}\right)$. Therefore, performing the ADIM in the WSS with knee flexion at $20^{\circ}$ must be more effective in increasing the TrA PCRs compared to other positions.

Park and Lee (2011) indicated that to perform the ADIM successfully, supine positions were more efficient than the WSS, and Manshadi et al. (2011) also indicated that while the ADIM was performed, TrA PCRs were significantly higher in supine positions (0.06) than in standing positions (0.02). However, unlike other functional positions, TrA PCRs in the WSS with knee flexion at $20^{\circ}$ were shown to be higher than those in supine positions shown in previous studies (Manshadi et al., 2011; Park and Lee, 2011), indicating that knee flexion at $20^{\circ}$ is an efficient position for performing successful ADIM.

The reason that the WSS with knee flexion at $20^{\circ}$ showed high $\operatorname{Tr}$ A PCR unlike other functional positions is that TrA CR was greatly affected by differences in ADIM methods. In the present study, the TrA PCR (0.05) that appeared while the ADIM was performed in the WSS with knee flexion at $0^{\circ}$ was shown to be higher than the result (0.03) of a previous study (Park and Lee, 2011) in which the ADIM was performed in the same position. The EO and IO+EO CRs were around 0.93 and 1.00. Therefore, the activities of the two superficially located muscles were minimized further than the present study but the TrA PCR was shown to be lower on the contrary.

Although there are differences between feedback devices, subjects were educated on the ADIM using feedback in both studies. However, in the present study, all the subjects used the ADIM method to contract the pelvic floor together, resulting in a CR of 1.58, higher than the TrA CR of 1.17 shown in the previous study (Park and Lee, 2011), in which the ADIM was performed in a conventional method. Therefore, knee flexion at $0^{\circ}$ in the present study showed much higher $\operatorname{Tr} A$ CRs than the previous study despite the fact that it showed higher EO and IO+EO CRs than the previous study, so that the TrA PCR was shown to be relatively higher. These results support the results of a previous study (Critchley, 2002) indicating that performing the ADIM along with the contraction of the pelvic floor can improve $\operatorname{Tr} \mathrm{A}$ activity, while suggesting that the effect can be maintained not only in four point kneeling positions but also in the WSS, which is a functional position.

The WSS with knee flexion at $20^{\circ}$ minimized $\mathrm{EO}$ and $\mathrm{IO}+\mathrm{EO}$ CRs with appropriate activity of quadriceps muscle while showing higher TrA CRs compared to other positions. As a result, this position showed the highest TrA PCR. Therefore, performing the ADIM in the WSS with knee flexion at $20^{\circ}$ appears to be the most appropriate position for TrA PCR.

\section{CONFLICT OF INTEREST}

No potential conflict of interest relevant to this article was reported.

\section{ACKNOWLEDGMENTS}

This work was supported by the Busan PNF Association.

\section{REFERENCES}

Ainscough-Potts AM, Morrissey MC, Critchley D. The response of the transverse abdominis and internal oblique muscles to different postures. Man Ther 2006;11:54-60.

Akuthota V, Nadler SF. Core strengthening. Arch Phys Med Rehabil 2004; 85:S86-S92.

Bjerkefors A, Ekblom MM, Josefsson K, Thorstensson A. Deep and superficial abdominal muscle activation during trunk stabilization exercises with and without instruction to hollow. Man Ther 2010;15:502-507.

Critchley D. Instructing pelvic floor contraction facilitates transversus abdominis thickness increase during low-abdominal hollowing. Physiother Res Int 2002;7:65-75.

Hodges PW, Richardson CA. Feedforward contraction of transversus abdominis is not influenced by the direction of arm movement. Exp Brain Res 1997;114:362-370.

Hubley-Kozey CL, Robbins SM, Rutherford DJ, Stanish WD. Reliability of surface electromyographic recordings during walking in individuals with knee osteoarthritis. J Electromyogr Kinesiol 2013;23:334-341.

Mannion AF, Pulkovski N, Gubler D, Gorelick M, O'Riordan D, Loupas T, Schenk P, Gerber H, Sprott H. Muscle thickness changes during abdominal hollowing: an assessment of between-day measurement error in controls and patients with chronic low back pain. Eur Spine J 2008; 17:494-501.

Manshadi FD, Parnianpour M, Sarrafzadeh J, Azghani MR, Kazemnejad A. Abdominal hollowing and lateral abdominal wall muscles' activity in both healthy men \& women: An ultrasonic assessment in supine 
and standing positions. J Bodyw Mov Ther 2011;15:108-113.

Mew R. Comparison of changes in abdominal muscle thickness between standing and crook lying during active abdominal hollowing using ultrasound imaging. Man Ther 2009;14:690-695.

Norris CM. Functional load abdominal training: part 2. Phys Ther Sport 2001;2:149-156.

O'Sullivan PB, Twomey L, Allison GT. Altered abdominal muscle recruitment in patients with chronic back pain following a specific exercise intervention. J Orthop Sports Phys Ther 1998;27:114-124.

O'Sullivan PB. Lumbar segmental 'instability': clinical presentation and specific stabilizing exercise management. Man Ther 2000;5:2-12.

Park DJ, Lee HO. Activation of abdominal Muscles during abdominal hollowing in four different positions. J Phys Ther Sci 2010;22:203-207.

Park DJ, Lee HO. The use of rehabilitative ultrasound imaging for feedback from the abdominal muscles during abdominal hollowing in different positions. J Phys Ther Sci 2011;23:895-898.

Park DJ, Lee SK. What is a Suitable Pressure for the Abdominal Drawing-in Maneuver in the Supine Position Using a Pressure Biofeedback Unit? J Phys Ther Sci. 2013;25:527-530.

Park KN, Cynn HS, Kwon OY, Lee WH, Ha SM, Kim SJ, Weon JH. Effects of the abdominal drawing-in maneuver on muscle activity, pelvic motions, and knee flexion during active prone knee flexion in patients with lumbar extension rotation syndrome. Arch Phys Med Rehabil
2011;92:1477-1483.

Richardson CA, Snijders CJ, Hides JA, Damen L, Pas MS, Storm J. The relation between the transversus abdominis muscles, sacroiliac joint mechanics, and low back pain. Spine 2002;27:399-405.

Rutherford DJ, Hubley-Kozey CL, Stanish WD. Maximal voluntary isometric contraction exercises: a methodological investigation in moderate knee osteoarthritis. J Electromyogr Kinesiol 2011;21:154-160.

Springer BA, Mielcarek BJ, Nesfield TK, Teyhen DS. Relationships among lateral abdominal muscles, gender, body mass index, and hand dominance. J Orthop Sports Phys Ther 2006;36: 289-297.

Stuge B, Laerum E, Kirkesola G, Vøllestad N. The efficacy of a treatment program focusing on specific stabilizing exercises for pelvic girdle pain after pregnancy: a randomized controlled trial. Spine 2004; 29:351-359.

Teyhen DS, Miltenberger CE, Deiters HM, Del Toro YM, Pulliam JN, Childs JD, Boyles RE, Flynn TW. The use of ultrasound imaging of the abdominal drawing-in maneuver in subjects with low back pain. J Orthop Sports Phys Ther 2005;35:346-355.

von Garnier K, Köveker K, Rackwitz B, Kober U, Wilke S, Ewert T, Stucki

G. Reliability of a test measuring transversus abdominis muscle recruitment with a pressure biofeedback unit. Physiotherapy 2009;95:814. 